\title{
Influence of peritoneal carcinomatosis on perioperative outcome in palliative gastric bypass for malignant gastric outlet obstruction - a retrospective cohort study
}

Jan Bednarsch ${ }^{1}$, Zoltan Czigany ${ }^{1}$, Daniel Heise ${ }^{1}$, Henning Zimmermann ${ }^{2}$, Joerg Boecker ${ }^{1}$, Tom Florian Ulmer ${ }^{1}$, Ulf Peter Neumann ${ }^{1,3}$ and Christian Klink ${ }^{1 *}$ (i)

\begin{abstract}
Background: Malignant gastric outlet obstruction (GOO) is commonly associated with the presence of peritoneal carcinomatosis (PC) and preferably treated by surgical gastrojejunostomy (GJJ) in patients with good performance. Here, we aim to investigate the role of PC as a risk factor for perioperative morbidity and mortality in patients with GOO undergoing GJJ.

Methods: Perioperative data of 72 patients with malignant GOO who underwent palliative GJJ at our institution between 2010 and 2019 were collected within an institutional database.

To compare perioperative outcomes of patients with and without PC, extensive group analyses were carried out.

Results: A set of 39 (54.2\%) patients was histologically diagnosed with concomitant PC while the remaining 33 (45.8\%) patients showed no clinical signs of PC. In-house mortality due to surgical complications was significantly higher in patients with PC $(9 / 39,23.1 \%)$ than in patients without PC $(2 / 33,6.1 \%, p=.046)$. Considerable differences were observed in terms of surgical complications such as anastomotic leakage rates $(2.8 \% \mathrm{vs} .0 \%, p=.187)$, delayed gastric emptying (33.3\% vs. $15.2 \%, p=.076)$, paralytic ileus ( $23.1 \%$ vs. $9.1 \%, p=.113)$, and pneumonia (17.9\% vs. $12.1 \%, p=.493$ ) without reaching the level of statistical significance.

Conclusions: $P C$ is an important predictor of perioperative morbidity and mortality patients undergoing GJJ for malignant GOO.
\end{abstract}

Keywords: Gastric outlet obstruction, Gastrojejujnostomy, Peritoneal carcinomatosis

\section{Background}

Malignant gastric outlet obstruction (GOO) is a common complication of advanced gastric, pancreatic, or biliary tumors as well as peritoneal carcinomatosis (PC) of various origin. The presence of GOO in patients with advanced cancer is associated with dehydration and malnutrition and significantly impacts the quality of life (QoL) due to symptoms like nausea, pain, weight loss, and recurrent vomiting $[1,2]$. This deterioration of the patient's general condition and performance often results in interruption of

\footnotetext{
*Correspondence: cklink@ukaachen.de

'Department of Surgery and Transplantation, University Hospital RWTH

Aachen, Pauwelsstrasse 30, 52074 Aachen, Germany

Full list of author information is available at the end of the article
}

systemic chemotherapy and requires rapid treatment to recover and continue medical therapy [3].

Surgical gastrojejunostomy (GJJ) has been considered as the mainstay of treatment for GOO [4]. However, in the last decade, endoscopic placement of a duodenal stent (DS) is becoming increasingly popular due to its simplicity and minimally invasive nature, leading to a faster recovery of oral intake and shorter hospital stay [5]. While DS is associated with better short-term outcomes, GJJ is preferable in patients with a longer life expectancy and good performance status since stentrelated complications (e.g., reocclusion and stent migration) do not occur and reinterventions are usually not necessary after GJJ [6]. Thus, GJJ is usually performed in

(c) The Author(s). 2020 Open Access This article is distributed under the terms of the Creative Commons Attribution 4.0 International License (http://creativecommons.org/licenses/by/4.0/), which permits unrestricted use, distribution, and 
case of GOO if the patient is intraoperatively diagnosed with $\mathrm{PC}$ or the primary tumor related to GOO is intraoperatively considered as technically not resectable [7].

PC is a late stage manifestation of several gastrointestinal malignancies characterized by tumor dissemination across the peritoneal cavity and frequently observed in patients with advanced gastric, hepatobiliary, or pancreatic cancer [8-10]. Patients with PC commonly present with symptoms such as nausea, abdominal pain or weight loss, and cachexia as the disease progresses over time $[11,12]$. Metachronous PC itself is also a prevalent cause of GOO in these patients.

Since PC represents an advanced stage of oncologic disease which is associated with malnutrition and impairment of the patients general condition, it seems plausible that the patients with $\mathrm{PC}$ are more prone to surgical complications and poor perioperative outcomes compared to individuals without PC [13]. Therefore, we here aim to investigate the role of $\mathrm{PC}$ as a risk factor for perioperative morbidity and mortality in patients with GOO undergoing GJJ.

\section{Methods}

\section{Patients}

Seventy-two $(n=72)$ consecutive patients with malignant gastric obstruction, who underwent palliative GJJ at the University Hospital RWTH Aachen (UH-RWTH) between 2010 and 2019, were included in this study. This retrospective study was conducted at the UHRWTH in accordance with the requirements of the Institutional Review Board of the RWTH Aachen University, the current version of the Declaration of Helsinki, and the good clinical practice guidelines (ICHGCP). Demographic characteristics are shown in Table 1.

\section{Clinical course and surgical technique}

All patients who were referred for surgical treatment to our institution underwent a detailed clinical work-up. Every patient included in this study presented with obstructive gastric outlet syndrome due to a malignant stenosis and was discussed in an interdisciplinary tumor board. The assessment of the patients' perioperative risk was based on the American Society of Anesthesiologists (ASA) score and the indication for surgery, and the selection of the operative procedure was made by an experienced visceral surgeon.

The majority of patients was diagnosed with an advanced tumor of hepatobiliary, pancreatic, duodenal, or gastric origin and underwent surgical exploration in curative intent. All patients presented with clinical symptoms of GOO preoperatively and showed signs of GOO in the preoperative cross-sectional imaging. If the local tumor was assessed as unresectable by the attending surgeon or if distant metastases (e.g., liver metastases or distant lymph nodes) and/or peritoneal carcinomatosis were diagnosed intraoperatively, a palliative gastrojejunostomy was carried out. In cases with significant biliary obstruction, an additional surgical hepaticojejunostomy was performed or it was resolved by percutaneous biliary drainage (PBD) during the postoperative course. The minority of patients underwent surgical exploration due to local recurrence of a previously resected malignant abdominal tumor and was also intraoperatively assessed as unresectable or showed distant metastases or PC. A detailed overview of the cohort and the particular surgical consideration for the GJJ is presented in Fig. 1.

Briefly, the surgical technique comprised an open hand-sewing technique with a side-to-side GJJ followed by a Roux-en-Y reconstruction with an end-to-side jejunojejunostomy. Whether a retrocolic or antecolic route for the GJJ was used was decided by the attending surgeon according to own preferences. The procedure was carried out laparoscopically in similar fashion with both anastomoses being realized by laparoscopic gastrointestinal staplers (Endo GIA, Medtronic, Dublin, Ireland).

The patients were treated postoperatively at a specialized intensive care unit or directly transferred to a normal postoperative ward based on the extent of the procedure and the individual preoperative medical condition. Parenteral nutrition was regularly used postoperatively if oral feeding could not be realized by the third postoperative day. No jejunostomy tubes were placed either intraoperatively or in the preoperative course in any patient of the cohort.

The presence of PC or distant metastases was intraoperatively indicated by fresh frozen sections and later confirmed by an experienced staff pathologist in the final pathological report in every case.

\section{Data collection}

All study data including demographics, tumor characteristics, clinical chemistry, and operative and postoperative data of every patient were retrospectively collected within an institutional database. The postoperative course was reviewed for in-house mortality as well as complications and rated by the Clavien-Dindo Classification [14]. Every patient's individual postoperative course was also assessed for specific surgical complications, e.g., anastomotic stenosis, anastomotic leakage, delayed gastric emptying (DGE), paralytic ileus, and pneumonia.

\section{Statistical analysis}

The primary endpoint of this study was the incidence of in-house mortality in patients undergoing palliative GJJ with and without PC. The secondary endpoints were 
Table 1 Patients' characteristics

\begin{tabular}{|c|c|c|c|c|}
\hline & \multirow{2}{*}{$\begin{array}{l}\text { Overall cohort } \\
(n=72)\end{array}$} & \multicolumn{3}{|c|}{ Peritoneal carcinomatosis vs. no peritoneal carcinomatosis (PC) } \\
\hline & & PC cohort $(n=39)$ & No PC cohort $(n=33)$ & $p$ value \\
\hline \multicolumn{5}{|l|}{ Demographics } \\
\hline Sex, $n(\%)$ & & & & .502 \\
\hline Male & $38(52.8)$ & $22(56.4)$ & $16(48.5)$ & \\
\hline Female & $34(47.2)$ & $17(43.6)$ & $17(51.5)$ & \\
\hline Age (years) & $66(58-74)$ & $66(57-74)$ & $65(59-74)$ & .591 \\
\hline $\mathrm{BMI}\left(\mathrm{kg} / \mathrm{m}^{2}\right)$ & $24(21-26)$ & $23(21-26)$ & $24(21-27)$ & .635 \\
\hline $\mathrm{ASA}, n(\%)$ & & & & .654 \\
\hline । & 0 & 0 & 0 & \\
\hline$\|$ & $19(26.4)$ & $12(30.8)$ & $7(21.2)$ & \\
\hline III & $45(62.5)$ & $23(59.0)$ & $22(66.7)$ & \\
\hline IV & $8(11.1)$ & $4(10.3)$ & $4(12.1)$ & \\
\hline V & 0 & & 0 & \\
\hline \multicolumn{5}{|l|}{ Tumor characteristics } \\
\hline Primary tumor, $n(\%)$ & & & & .324 \\
\hline Pancreatic adenocarcinoma & $42(58.3)$ & $18(46.2)$ & $24(72.7)$ & \\
\hline Cholangiocellular carcinoma & $10(13.9)$ & $8(20.5)$ & $2(6.1)$ & \\
\hline Gastric adenocarcinoma & $5(6.9)$ & $3(7.7)$ & $2(6,1)$ & \\
\hline Intestinal carcinoma & $3(4.2)$ & $2(5.1)$ & $1(3.0)$ & \\
\hline Colorectal carcinoma & $6(8.3)$ & $4(10.3)$ & $2(6.1)$ & \\
\hline Renal cell carcinoma & $2(2.8)$ & $1(2.6)$ & $1(3.0)$ & \\
\hline Transitional cell carcinoma & $1(1.4)$ & $1(2.6)$ & 0 & \\
\hline Mammarian carcinoma & $2(2.8)$ & $2(5.1)$ & 0 & \\
\hline Ovarian cancer & $1(1.4)$ & 0 & $1(3.0)$ & \\
\hline \multicolumn{5}{|l|}{ Synchronous/metachronous PC, n (\%) } \\
\hline Synchronous PC & n.a. & $20(51.3)$ & n.a. & \\
\hline Metachronous PC & n.a. & $19(48.7)$ & n.a. & \\
\hline Distant metastasis, $n$ (\%) & & & & .132 \\
\hline Yes & $24(33.3)$ & $10(25.6)$ & $14(42.4)$ & \\
\hline No & $48(66.7)$ & $29(74.4)$ & $19(57.6)$ & \\
\hline Preoperative chemotherapy, $n(\%)$ & & & & .776 \\
\hline Yes & $10(13.9)$ & $5(12.8)$ & $5(15.2)$ & \\
\hline No & $62(86.1)$ & $34(87.2)$ & $28(84.8)$ & \\
\hline \multicolumn{5}{|l|}{ Clinical chemistry } \\
\hline Sodium (mmol/l) & $139(136-141)$ & $139(136-142)$ & $138(136-141)$ & .536 \\
\hline Hemoglobin (g/dl) & $10.6(9.3-12.1)$ & $11.2(9.0-12.7)$ & $10.2(9.4-11.7)$ & .235 \\
\hline Platelet count (nl) & $242(198-355)$ & $250(201-341)$ & $215(195-416)$ & .739 \\
\hline Total bilirubin (mg/dl) & $0.6(0.4-2.0)$ & $0.59(0.37-1.42)$ & $0.76(0.44-3.81)$ & .235 \\
\hline Prothrombin time (\%) & 87 (77-98) & $89(76-98)$ & $85(76-98)$ & .773 \\
\hline INR & $1.1(1.0-1.2)$ & $1.1(1.0-1.2)$ & $1.1(1.0-1.2)$ & .874 \\
\hline Albumin (g/l) & $32(29-39)$ & $34(28-38)$ & $31(28-39)$ & .394 \\
\hline Creatinine (mg/dl) & $0.8(0.6-1.0)$ & $0.9(0.6-1.1)$ & $0.7(0.6-0.9)$ & .230 \\
\hline CRP $(\mathrm{mg} / \mathrm{l})$ & $29(11-56)$ & $25(8-51)$ & $31(13-81)$ & .317 \\
\hline
\end{tabular}


Table 1 Patients' characteristics (Continued)

\begin{tabular}{|c|c|c|c|c|}
\hline & \multirow{2}{*}{$\begin{array}{l}\text { Overall cohort } \\
(n=72)\end{array}$} & \multicolumn{3}{|c|}{ Peritoneal carcinomatosis vs. no peritoneal carcinomatosis (PC) } \\
\hline & & PC cohort $(n=39)$ & No PC cohort $(n=33)$ & $p$ value \\
\hline \multicolumn{5}{|l|}{ Operative data } \\
\hline Laparoscopic surgery, $n(\%)$ & $2(2.8)$ & 0 & $2(6.1)$ & .119 \\
\hline Concomitant hepaticojejunostomy, $n$ (\%) & $26(36.1)$ & $10(25.6)$ & $16(48.5)$ & .044 \\
\hline Operative procedure, $n(\%)$ & & & & .831 \\
\hline Antecolic approach & $6(8.3)$ & $3(7.7)$ & $3(9.1)$ & \\
\hline Retrocolic approach & $66(91.7)$ & $36(92.3)$ & $30(90.9)$ & \\
\hline Operation time (min) & $170(128-214)$ & $160(113-215)$ & $192(150-216)$ & .124 \\
\hline Intraoperative blood transfusion $(U)$ & 0 & 0 & $0(0-2)$ & .817 \\
\hline Intraoperative fresh frozen plasma $(U)$ & 0 & 0 & 0 & .108 \\
\hline \multicolumn{5}{|l|}{ Postoperative data } \\
\hline Intensive care, days & $1(0-2)$ & $1(0-2)$ & $1(0-3)$ & .240 \\
\hline Hospitalization, days & $13(9-18)$ & $13(10-20)$ & $12(9-17)$ & .392 \\
\hline $\mathrm{PBD}^{*}, n(\%)$ & $6(13.0)$ & $5(17.2)$ & $1(5.9)$ & .270 \\
\hline Postoperative complications, $n$ (\%) & & & & .381 \\
\hline No complications & $23(31.9)$ & $13(33.3)$ & $10(30.3)$ & \\
\hline Clavien-Dindo I & $8(11.1)$ & $4(10.3)$ & $4(12.1)$ & \\
\hline Clavien-Dindo ॥ & $20(27.8)$ & $8(20.5)$ & $12(36.4)$ & \\
\hline Clavien-Dindo IIla & $4(5.6)$ & $2(5.1)$ & $2(6.1)$ & \\
\hline Clavien-Dindo IIIb & $5(6.9)$ & $2(5.1)$ & $3(9.1)$ & \\
\hline Clavien-Dindo IVa & $1(1.4)$ & $1(2.6)$ & 0 & \\
\hline Clavien-Dindo IVb & 0 & 0 & 0 & \\
\hline Clavien-Dindo V & $11(15.3)$ & $9(23.1)$ & $2(6.1)$ & \\
\hline In-house mortality, n (\%) & $11(15.3)$ & $9(23.1)$ & $2(6.1)$ & .046 \\
\hline Anastomotic stenosis, $n(\%)$ & $2(2.8)$ & $1(2.6)$ & $1(3)$ & .905 \\
\hline Anastomotic leakage, $n(\%)$ & $2(2.8)$ & $2(5.1)$ & 0 & .187 \\
\hline Delayed gastric emptying, $n(\%)$ & $18(25.0)$ & $13(33.3)$ & $5(15.2)$ & .076 \\
\hline Paralytic ileus, $n(\%)$ & $12(16.7)$ & $9(23.1)$ & $3(9.1)$ & .113 \\
\hline Pneumonia, $n(\%)$ & $11(15.3)$ & $7(17.9)$ & $4(12.1)$ & .493 \\
\hline
\end{tabular}

Data presented as median and interquartile range if not noted otherwise. Categorical data were compared using the chi-squared test, Fisher's exact test, or linearby-linear association according to the scale and number of cases. Data derived from continuous variables of different groups were compared by the MannWhitney $U$ test

$C R P$ c-reactive protein, INR international normalized ratio, $P B D$ percutaneous biliary drainage, $P C$ peritoneal carcinomatosis

* Statistics are calculated for patients who did not undergo concomitant hepaticojejunostomy during the surgical procedure

perioperative complications, duration of hospitalization, and ICU stay. Categorical data are presented as counts and percentages, compared using the chi-squared test, Fisher's exact test, or linear-by-linear association according to the scale and number of cases. Data derived from continuous variables are presented as median and interquartile range and are analyzed by the Mann-Whitney $U$ test. Associations between pre- and intraoperative variables and postoperative mortality were assessed by means of binary logistic regression. The level of significance was set to $p<0.05$, and $p$ values are given for two-sided testing. Analyses were performed using SPSS Statistics 24 (IBM Corp., Armonk, NY, USA).

\section{Results}

\section{Patient cohort}

A total of 72 patients underwent palliative GJJ due to malignant GOO at our institution from 2010 to 2019. A subset of $39(54.2 \%)$ patients was histologically diagnosed with concomitant PC while the remaining 33 (45.8\%) patients showed no clinical signs of PC.

\section{Preoperative, intraoperative, and postoperative data}

The overall cohort comprised 38 (52.8\%) male and 34 (47.2\%) female patients with a median age of 66 years who were mostly assessed ASA III or higher (53/72, 73.6\%). The majority of patients was diagnosed with 

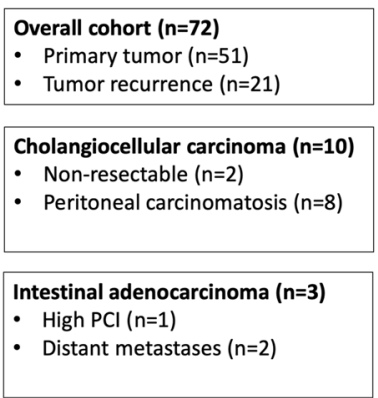

Ovarian cancer $(n=1)$

- Distant metastases $(n=1)$

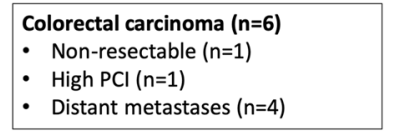

Renal cell carcinoma $(n=2)$

- Non-resectable $(n=1)$

- Peritoneal carcinomatosis $(n=1)$

Transitional cell carcinoma $(n=1)$

- Peritoneal carcinomatosis $(n=1)$

Fig. 1 Overall cohort of patients undergoing gastric outlet obstruction. The overall cohorts comprised patients with pancreatic, cholangiocellular, gastric, intestinal, colorectal, renal cell, transitional cell, and mammarian carcinoma as well as ovarian cancer. Surgical gastrojejunostomy instead of curative surgery was considered in cases with technically non-resectable cancer (pancreatic, cholangiocellular, intestinal, colorectal, and renal cell carcinoma), distant metastases (pancreatic, cholangiocellular, gastric, and colorectal carcinoma and ovarian cancer), peritoneal carcinomatosis presenting with a high $\mathrm{PCl}$ in tumor entities qualifying for cytoreductive surgery and HIPEC (gastric, intestinal, and colorectal carcinoma) as well as cases presenting with peritoneal carcinomatosis tumor entities which preclude curative surgery (pancreatic, cholangiocellular, renal cell, transitional cell, and mammarian carcinoma). HIPEC, hyperthermic intraperitoneal chemotherapy; PCl, peritoneal carcinomatosis index

pancreatic adenocarcinoma $(42 / 72,58.3 \%)$ followed by cholangiocellular carcinoma (10/72, 13.9\%), colorectal cancer $(6 / 72,8.3 \%)$, and gastric adenocarcinoma $(5 / 72$, $6.9 \%)$. Only a subset of patients was treated with chemotherapy prior to surgery $(10 / 72,13.9 \%)$. No statistical difference was observed between patients with and without PC with respect to demographics and tumor characteristics, e.g., the primary diagnosis $(p=.324)$, the presence of distant metastases $(p=.132)$, or the utilization of preoperative chemotherapy ( $p=.776)$.

Laparoscopic surgery was seldomly applied in the cohort $(2 / 72,2.8 \%)$, and the gastrojejunostomy was mostly carried out in a retrocolic technique $(66 / 72,91.7 \%)$ with no difference between patients with and without PC ( $p=$ .831). Also, the median operating time showed no difference in patients with and without PC (160 min vs. 192 min, $p=.124)$. However, an additional hepaticojejunostomy was more common in patients without PC (16/33, $48.5 \%)$ than in patients with PC $(10 / 39,25.6 \%, p=.044)$.

No difference was observed in the median duration of hospitalization (13 vs. 12 days, $p=.392$ ) and intensive care treatment ( 1 vs. 1 day, $p=.240$ ) between patients with and without PC. In-house mortality due to surgical complications was significantly higher in patients with PC $(9 / 39,23.1 \%)$ than in patients without PC $(2 / 33$, $6.1 \%, p=.046)$. Surgery-specific complications such as anastomotic leakage $(2 / 39(2.8 \%)$ vs. $0 / 33,(0 \%), p=$ $.187)$, DGE (13/39 (33.3\%) vs. 5/33 (15.2\%), $p=.076)$, paralytic ileus (9/39 (23.1\%) vs. $3 / 33(9.1 \%), p=.113)$, and pneumonia $(7 / 39(17.9 \%)$ vs. $4 / 33(12.1 \%), p=.493)$ were tendentially higher in patients with $\mathrm{PC}$ than in patients without PC but did not achieve a statistically significant differences between the groups.
A univariate binary logistic regression was carried out for postoperative mortality including all available pre- and intraoperative variables for patients with PC (Table 2). Here, no statistical significance was observed between pre- and intraoperative characteristics and postoperative mortality.

More details regarding perioperative characteristics and group comparisons are presented in Table 1.

\section{Discussion}

Since traditional imaging such as classic computed tomography $(\mathrm{CT})$ and magnetic resonance imaging (MRI) as well as positron-emission tomography (PET) lacks sensitivity to preoperatively detect $\mathrm{PC}$, patients with $\mathrm{PC}$ and GOO often undergo surgical exploration with curative intent [15-17]. However, if $\mathrm{PC}$ is subsequently diagnosed intraoperatively, a GJJ is often performed to treat GOO without delay.

Here, we aimed to evaluate the effects of PC on perioperative outcomes in patients who underwent GJJ for malignant GOO. Therefore, we investigated the incidence of postoperative complications in terms of total and surgery-specific complications in these particular patients. Our statistical group comparison showed that surgeryrelated in-house mortality was significantly higher in patients with PC (23.1\%) than in patients without PC (6.1\%). Furthermore, surgery-specific complications such as anastomotic leakage, DGE, paralytic ileus, and pneumonia appeared to be more common in the PC group than in the non-PC group.

The reason for this observation remains speculative. While the analyzed surgery-specific complications did not show statistical significance, each complication occurred numerically higher in the PC cohort which might 
Table 2 Univariable binary logistic regression of postoperative mortality in patients with peritoneal carcinomatosis undergoing gastrojejunostomy

\begin{tabular}{|c|c|c|}
\hline \multirow[t]{2}{*}{ Variables } & \multicolumn{2}{|c|}{ In-house mortality } \\
\hline & $\operatorname{Exp}(\mathrm{B}) / \mathrm{HR}$ & $p$ value \\
\hline Sex $($ male $=1)$ & 0.571 & .482 \\
\hline Age & 1.072 & .137 \\
\hline BMI & 1.083 & .349 \\
\hline ASA scale & 3.309 & .090 \\
\hline \multicolumn{3}{|l|}{ Primary tumor } \\
\hline Pancreatic adenocarcinoma & 1 & \\
\hline Other primary tumors & 0.612 & .521 \\
\hline \multicolumn{3}{|l|}{ Synchronous/metachronous PC } \\
\hline Synchronous PC & 0.438 & .299 \\
\hline Metachronous PC & 1 & \\
\hline Distant metastasis (no. $=1$ ) & 0.786 & .789 \\
\hline Preoperative chemotherapy $(\mathrm{no} .=1)$ & 2.571 & .348 \\
\hline Sodium & 0.996 & .963 \\
\hline Hemoglobin & 0.824 & .350 \\
\hline Platelet count & 1.000 & .977 \\
\hline Total bilirubin & 0.989 & .954 \\
\hline Prothrombin time & 0.973 & .267 \\
\hline INR & 20.409 & .211 \\
\hline Albumin & 0.946 & .348 \\
\hline Creatinine & 1.047 & .967 \\
\hline CRP & 1.009 & .418 \\
\hline Laparoscopic surgery (no. = 1) & n.a. & n.a. \\
\hline Concomitant hepaticojejunostomy (no. = 1) & 0.292 & .277 \\
\hline \multicolumn{3}{|l|}{ Operative procedure } \\
\hline Antecolic approach & 0.000 & .999 \\
\hline Retrocolic approach & 1 & \\
\hline Operation time (min) & 1.000 & .975 \\
\hline Intraoperative blood transfusion $($ no. $=1$ ) & 0.625 & .687 \\
\hline Intraoperative fresh frozen plasma (no. = 1) & 0.000 & .999 \\
\hline
\end{tabular}

Various parameters are tested for association with in-house mortality ASA American Society of Anesthesiologists classification, $B M I$ body mass index, $H R$ ratio, INR international normalized ratio

have translated into the observed increased overall mortality rate after surgery. Another possible explanation might be subclinical intestinal obstruction distal to the GJJ which causes intestinal congestion and subsequently anastomotic problems or aspiration [18]. Also, malnutrition is a major problem in patients with $\mathrm{PC}$ and has been in the focus of research in the last decade resulting in its identification as an important predictor for postoperative complications in abdominal and extra-abdominal surgery [19-21]. In addition to malnutrition, tumor cachexia, a complex multifactorial condition that arises from a combination of metabolic alterations, systemic inflammation, and decreased appetite, is also a major concern in patients with increased tumor burden [22]. Tumor cachexia is directly associated with malnutrition and associated with impaired wound healing, increased risk for surgical complications, and impaired overall outcome [23, 24].

Our patients display an in-house mortality of $15.3 \%$ in the overall cohort with $23.1 \%$ mortality in patients with PC and 6.1\% without PC. This mortality was based on anastomotic leakage in two individuals, while most of the other patients with fatal outcome presented with postoperative ileus and subsequently developed abdominal sepsis or severe pneumonia presumably due to aspiration. The reported in-house mortality might appear high for gastrointestinal surgery. However, reported mortality rates after GJJ for unresectable cancer range from 3 to $30 \%$ in literature $[9,25-29]$ supporting the validity of our data. Interestingly, the presence of PC as a risk factor for surgical outcome has not been directly investigated in previous reports. While previous literature, comprising various cohorts from the last couple of decades, focuses mostly on general outcome figures, the report of Poulsen et al. analyzed surgical outcomes of 165 patients of which 120 individuals presented with malignant and 45 individuals with benign GOO and conducted a detailed analysis of surgical morbidity and mortality [25]. In this paper, the observed 30-day mortality in case of malignant GOO was $29 \%$ with age, comorbidities, hypoalbuminemia, and hyponatremia being the major drivers of mortality in multivariate analysis. Unfortunately, the role of peritoneal carcinomatosis was also not investigated in this particular work, but it illustrates the importance of patient-related characteristics as a risk factor.

Of note in this context, the performance of a concomitant hepaticojejunostomy during the surgical procedure was significantly more frequent in our non-PC cohort (48.5\%) than in our PC cohort $(24.6 \%, p=.044)$. However, this additional procedure did obviously not translate into an increased risk for surgical complications. This particular finding, as well as the increase of mortality by peritoneal carcinomatosis as suggested by our data and the results of Poulsen et al., indicates a superiority of patientand tumor-related characteristics over the surgical technique itself in the perioperative risk assessment for patients undergoing GJJ for malignant GOO [25].

While a mortality of $6.1 \%$ in patients without PC does encourage the utilization of palliative GJJ in these particular patients, the observed $23.1 \%$ mortality in patients with PC combined with the reported perioperative outcomes in the literature does demand a critical discussion of therapeutic alternatives in this subcohort. DS has evolved as a viable option for patients with malignant GOO and limited life expectancy [5]. DS is effective and 
less invasive compared with surgical GJJ but has been reported to be associated with higher rates of reintervention and recurrent obstructive symptoms reported [5, 30, 31]. However, it is debatable whether potential reinterventions on the long run are favorable compared to an increased perioperative mortality in patients with PC who per se have a reduced life expectancy [32, 33]. A recent report of Park et al. does investigate the role of PC and malignant ascites in gastric cancer [34]. The experienced group from South Korea observed a longer patency after GJJ compared to DS in patients with PC alone and in patients with PC and ascites as well as longer overall survival after GJJ in patients with $P C$ and ascites. Anyhow, these superior results for GJJ might not be applicable to PC of other origins which are less responsive to palliative chemotherapy, e.g., hepatobiliary or pancreatic cancer. Another comparative therapy currently investigated is endoscopic ultrasound-guided GJJ (EUS-GJJ) which warrants further research [35]. From a pure surgical point of view, laparoscopic GJJ may provide an improvement over open GJJ but the currently available literature mainly consists of small case series or small case-control studies and 1 randomized trial comparing open and laparoscopic GJJ in only 24 patients $[36,37]$. Given the limited data and the complexity of malignant GOO, more clinical evidence is needed to evaluate the potential benefits of the laparoscopic approach over conventional open surgery [36].

In summary, $\mathrm{PC}$ appears to be a major driver of complications and dismal outcome in GJJ. Our findings warrant further investigations to explore the exact role of PC on clinical outcomes in the palliative treatment of GOO. Unfortunately, we were not able to determine statistically significant predictors of impaired postoperative outcome in patients with PC within our small retrospective cohort. Thus, prospective clinical trials with adequate sample sizes stratifying data or treatment by the presence of PC are warranted.

Some obvious limitations of our retrospective study need to be considered when interpreting the results. Firstly, the data represent a retrospective single-center experience, reflecting our individual approach and surgical technique in GOO. Secondly, our cohort shows a considerable heterogeneity in terms of primary tumors and no separate analyses for each primary tumor were conducted. This might be of a major importance since some of the tumor entities are commonly known to be more aggressive, e.g., cholangiocellular carcinoma, than others. We particularly decided to include various primary tumors to gain sample size and strengthen our statistical analysis. Also, all demographics including the primary tumor and perioperative characteristics usually associated with perioperative outcome showed no statistical difference between patients with and without PC supporting the validity of our findings. However, it has to be taken into account that we are not able to correlate our findings with estimates of the severity of the carcinomatosis, e.g., peritoneal carcinomatosis index (PCI), due to the retrospective nature of the study. Thirdly, we were not able to report the outcome in benign GOO and are not able to compare our results with DS in case of malignant GOO as a comparative treatment. Anyhow, our aim was to investigate the role of $\mathrm{PC}$ as a potential risk factor for surgical complications in patients who undergo GJJ, since in clinical reality, GJJ is more common than DS in patients who are surgically explored for a curativeintent resection and are intraoperatively diagnosed with PC or considered to be technically not resectable.

\section{Conclusions}

Notwithstanding the aforementioned limitations, we have identified PC as an important predictor of perioperative morbidity and mortality in a cohort of patients undergoing GJJ for malignant GOO. Larger multicentric studies are warranted to confirm and validate these findings.

\section{Abbreviations}

ASA: American Society of Anesthesiologists; BMI: Body mass index; CRP: Creactive protein; CT: Computed tomography; DGE: Delayed gastric emptying; DS: Duodenal stent; EBD: Endoscopic biliary drainage; EUS-GJJ: Endoscopic ultrasound-guided gastrojejunostomy; GJJ: Gastrojejunostomy; GOO: Gastric outlet obstruction; HIPEC: Hyperthermic intraperitoneal chemotherapy; MRI: Magnetic resonance imaging; PBD: Percutaneous biliary drainage; PC: Peritoneal carcinomatosis; PCl: Peritoneal carcinomatosis index; PET: Positron-emission tomography; QoL: Quality of life; RWTH: RheinischWestfälische Technische Hochschule; UICC: Union for International Cancer Control

\section{Acknowledgements}

Not applicable.

\section{Authors' contributions}

JBe, UPN, and CK designed the study. JBe, ZC, and JBo collected the data. $\mathrm{JBe}, \mathrm{DH}, \mathrm{HZ}, \mathrm{TFU}$, and CK analyzed and interpreted the data. JBe and CK drafted the manuscript. $\mathrm{ZC}, \mathrm{DH}, \mathrm{HZ}, \mathrm{JBO}, \mathrm{TFU}$, and UPN critically revised the manuscript. All authors read and approved the final manuscript.

\section{Funding}

No particular funding was received for this research paper.

\section{Availability of data and materials}

The datasets used and/or analyzed during the current study are available from the corresponding author on reasonable request.

\section{Ethics approval and consent to participate}

The need for ethics approval and consent was waived by the Institutional Review Board of the RWTH Aachen University due to the retrospective nature of the study (EK 312/19).

\section{Consent for publication}

Not applicable.

\section{Competing interests}

The authors declare that they have no competing interests.

\section{Author details}

${ }^{1}$ Department of Surgery and Transplantation, University Hospital RWTH Aachen, Pauwelsstrasse 30, 52074 Aachen, Germany. ${ }^{2}$ Department of Internal Medicine III, University Hospital RWTH Aachen, Pauwelsstrasse 30, 52074 
Aachen, Germany. ${ }^{3}$ Department of Surgery, Maastricht University Medical Centre (MUMC), P. Debyelaan 25, 6229 HX, Maastricht, Netherlands.

\section{Received: 20 November 2019 Accepted: 23 January 2020} Published online: 31 January 2020

\section{References}

1. Del Piano M, Ballare M, Montino F, Todesco A, Orsello M, Magnani C, Garello E. Endoscopy or surgery for malignant Gl outlet obstruction? Gastrointest Endosc. 2005;61:421-6

2. Keranen I, Kylanpaa L, Udd M, Louhimo J, Lepisto A, Halttunen J, Kokkola A. Gastric outlet obstruction in gastric cancer: a comparison of three palliative methods. J Surg Oncol. 2013;108:537-41.

3. Lillemoe KD, Cameron JL, Hardacre JM, Sohn TA, Sauter PK, Coleman J, Pitt HA, Yeo CJ. Is prophylactic gastrojejunostomy indicated for unresectable periampullary cancer? A prospective randomized trial. Ann Surg. 1999;230: 322-8 discussion 328-330.

4. Ly J, O'Grady G, Mittal A, Plank L, Windsor JA. A systematic review of methods to palliate malignant gastric outlet obstruction. Surg Endosc. 2010; 24:290-7

5. Fiori E, Lamazza A, Demasi E, Decesare A, Schillaci A, Sterpetti AV. Endoscopic stenting for gastric outlet obstruction in patients with unresectable antro pyloric cancer. Systematic review of the literature and final results of a prospective study. The point of view of a surgical group. Am J Surg. 2013;206:210-7.

6. Bian SB, Shen WS, Xi HQ, Wei B, Chen L. Palliative therapy for gastric outlet obstruction caused by unresectable gastric cancer: a meta-analysis comparison of gastrojejunostomy with endoscopic stenting. Chin Med J (Engl). 2016;129:1113-21.

7. Kneuertz PJ, Cunningham SC, Cameron JL, Torrez S, Tapazoglou N, Herman JM, Makary MA, Eckhauser F, Wang J, Hirose K, et al. Palliative surgical management of patients with unresectable pancreatic adenocarcinoma: trends and lessons learned from a large, single institution experience. J Gastrointest Surg. 2011;15:1917-27.

8. Coccolini F, Gheza F, Lotti M, Virzi S, lusco D, Ghermandi C, Melotti R, Baiocchi G, Giulini SM, Ansaloni L, Catena F. Peritoneal carcinomatosis. World J Gastroenterol. 2013;19:6979-94.

9. Mann CD, Thomasset SC, Johnson NA, Garcea G, Neal CP, Dennison AR, Berry DP. Combined biliary and gastric bypass procedures as effective palliation for unresectable malignant disease. ANZ J Surg. 2009;79:471-5.

10. Lambert LA, Wiseman J. Palliative management of peritoneal metastases. Ann Surg Oncol. 2018;25:2165-71.

11. McMullen JRW, Selleck M, Wall NR, Senthil M. Peritoneal carcinomatosis: limits of diagnosis and the case for liquid biopsy. Oncotarget. 2017:8:43481-90.

12. Low RN, Barone RM, Rousset P. Peritoneal MRI in patients undergoing cytoreductive surgery and HIPEC: history, clinical applications, and implementation. Eur J Surg Oncol. 2019. [Online ahead of print]

13. Levy AD, Shaw JC, Sobin LH. Secondary tumors and tumorlike lesions of the peritoneal cavity: imaging features with pathologic correlation. Radiographics. 2009;29:347-73.

14. Dindo D, Demartines N, Clavien PA. Classification of surgical complications: a new proposal with evaluation in a cohort of 6336 patients and results of a survey. Ann Surg. 2004;240:205-13.

15. Esquivel J, Chua TC, Stojadinovic A, Melero JT, Levine EA, Gutman M, Howard R, Piso P, Nissan A, Gomez-Portilla A, et al. Accuracy and clinical relevance of computed tomography scan interpretation of peritoneal cancer index in colorectal cancer peritoneal carcinomatosis: a multiinstitutional study. J Surg Oncol. 2010;102:565-70.

16. Fehniger J, Thomas S, Lengyel E, Liao C, Tenney M, Oto A, Yamada SD. A prospective study evaluating diffusion weighted magnetic resonance imaging (DW-MRI) in the detection of peritoneal carcinomatosis in suspected gynecologic malignancies. Gynecol Oncol. 2016;142:169-75.

17. Klumpp BD, Aschoff P, Schwenzer N, Fenchel M, Koenigsrainer I, Falch C, Bruecher B, Claussen CD, Koenigsrainer A, Pfannenberg C, et al. Peritoneal carcinomatosis: comparison of dynamic contrast-enhanced magnetic resonance imaging with surgical and histopathologic findings. Abdom Imaging. 2012;37:834-42

18. Sadeghi B, Arvieux C, Glehen O, Beaujard AC, Rivoire M, Baulieux J, Fontaumard E, Brachet A, Caillot JL, Faure JL, et al. Peritoneal carcinomatosis from non-gynecologic malignancies: results of the EVOCAPE 1 multicentric prospective study. Cancer. 2000;88:358-63.
19. Aria Guerra E, Cortes-Salgado A, Mateo-Lobo R, Nattero L, Riveiro J, VegaPinero B, Valbuena B, Carabana F, Carrero C, Grande E, et al. Role of parenteral nutrition in oncologic patients with intestinal occlusion and peritoneal carcinomatosis. Nutr Hosp. 2015;32:1222-7.

20. Wilson JM, Holzgrefe RE, Staley CA, Schenker ML, Meals C. The effect of malnutrition on postoperative complications following surgery for distal radius fractures. J Hand Surg Am. 2019;44:742-50.

21. Weimann A, Braga M, Carli F, Higashiguchi T, Hubner M, Klek S, Laviano A Ljungqvist O, Lobo DN, Martindale R, et al. ESPEN guideline: clinical nutrition in surgery. Clin Nutr. 2017:36:623-50.

22. Fearon K, Strasser F, Anker SD, Bosaeus I, Bruera E, Fainsinger RL, Jatoi A Loprinzi C, MacDonald N, Mantovani G, et al. Definition and classification of cancer cachexia: an international consensus. Lancet Oncol. 2011;12:489-95.

23. Mantzorou M, Koutelidakis A, Theocharis S, Giaginis C. Clinical value of nutritional status in cancer: what is its impact and how it affects disease progression and prognosis? Nutr Cancer. 2017;69:1151-76.

24. Belghiti J, Langonnet F, Bourstyn E, Fekete F. Surgical implications of malnutrition and immunodeficiency in patients with carcinoma of the oesophagus. Br J Surg. 1983;70:339-41.

25. Poulsen M, Trezza M, Atimash GH, Sorensen LT, Kallehave F, Hemmingsen U, Jorgensen LN. Risk factors for morbidity and mortality following gastroenterostomy. J Gastrointest Surg. 2009;13:1238-44.

26. Ouchi K, Sugawara T, Ono H, Fujiya T, Kamiyama Y, Kakugawa Y, Mikuni J, Yamanami $\mathrm{H}$. Therapeutic significance of palliative operations for gastric cancer for survival and quality of life. J Surg Oncol. 1998;69:41-4.

27. La Ferla G, Murray WR. Carcinoma of the head of the pancreas: bypass surgery in unresectable disease. Br J Surg. 1987;74:212-3.

28. Singh SM, Reber HA. Surgical palliation for pancreatic cancer. Surg Clin North Am. 1989;69:599-611.

29. Schantz SP, Schickler W, Evans TK, Coffey RJ. Palliative gastroenterostomy for pancreatic cancer. Am J Surg. 1984;147:793-6.

30. Khashab M, Alawad AS, Shin EJ, Kim K, Bourdel N, Singh VK, Lennon AM, Hutfless S, Sharaiha RZ, Amateau S, et al. Enteral stenting versus gastrojejunostomy for palliation of malignant gastric outlet obstruction. Surg Endosc. 2013;27:2068-75.

31. Rudolph HU, Post S, Schluter M, Seitz U, Soehendra N, Kahler G. Malignant gastroduodenal obstruction: retrospective comparison of endoscopic and surgical palliative therapy. Scand J Gastroenterol. 2011;46:583-90.

32. Thomassen I, Lemmens VE, Nienhuijs SW, Luyer MD, Klaver YL, de Hingh $\mathbb{H}$. Incidence, prognosis, and possible treatment strategies of peritoneal carcinomatosis of pancreatic origin: a population-based study. Pancreas. 2013:42:72-5

33. Takahara N, Isayama H, Nakai Y, Sasaki T, Saito K, Hamada T, Mizuno S, Miyabayashi K, Mohri D, Kogure $H$, et al. Pancreatic cancer with malignant ascites: clinical features and outcomes. Pancreas. 2015:44:380-5.

34. Park CH, Park JC, Kim EH, Chung H, An JY, Kim HI, Shin SK, Lee SK, Cheong $J H$, Hyung WJ, et al. Impact of carcinomatosis and ascites status on longterm outcomes of palliative treatment for patients with gastric outlet obstruction caused by unresectable gastric cancer: stent placement versus palliative gastrojejunostomy. Gastrointest Endosc. 2015;81:321-32.

35. Perez-Miranda M, Tyberg A, Poletto D, Toscano E, Gaidhane M, Desai AP, Kumta NA, Fayad L, Nieto J, Barthet M, et al. EUS-guided gastrojejunostomy versus laparoscopic gastrojejunostomy: an international collaborative study. J Clin Gastroenterol. 2017;51:896-9.

36. Manuel-Vazquez A, Latorre-Fragua R, Ramiro-Perez C, Lopez-Marcano A, la Plaza-Llamas R, Ramia JM. Laparoscopic gastrojejunostomy for gastric outlet obstruction in patients with unresectable hepatopancreatobiliary cancers: a personal series and systematic review of the literature. World J Gastroenterol. 2018;24:1978-88.

37. Navarra G, Musolino C, Venneri A, De Marco ML, Bartolotta M. Palliative antecolic isoperistaltic gastrojejunostomy: a randomized controlled trial comparing open and laparoscopic approaches. Surg Endosc. 2006;20:1831-4.

\section{Publisher's Note}

Springer Nature remains neutral with regard to jurisdictional claims in published maps and institutional affiliations. 